\title{
Addressing skewed sex ratio at birth in China: practices and challenges
}

\section{Mengjun Tang ${ }^{1}$}

Received: 10 December 2020 / Accepted: 15 December 2020 / Published online: 4 February 2021

(c) The Author(s) 2021

\begin{abstract}
The sex ratio at birth (SRB) in China began to rise rapidly in the 1980s and declined after 2009. The ratio has maintained a downward trend since 2009, but is still higher than the normal level. The government of China has committed to redress the distortions in SRB and has initiated multiple actions to accomplish that end. Against a background of economic development, accelerating modernization and the empowerment of women, China has strengthened governance and made significant progress in balancing the sex ratio at birth. This paper examines the trend for SRB in China, summarizes the practices that address gender imbalance at birth, and analyzes the challenges ahead.
\end{abstract}

Keywords Sex ratio at birth $\cdot$ Governance $\cdot$ Gender equality

\section{Introduction}

Sex ratio at birth (SRB) refers to the ratio between live male births and live female births during a certain period of time. The normal range is between 103 to 107 males and 100 females. Not a simple demographic indicator, SRB determines the gender structure of a population in the future, and is also a key indicator of gender equality. A high SRB is considered a symptom of pervasive social, cultural, political, and economic injustices against women, infringing upon women's rights to survive and develop.

At present, more than a dozen countries in the world are experiencing distorted SRB. These countries are in East, West and South Asia, and Eastern Europe; the affected population accounts for about $40 \%$ of the world's population. China is the world's most populous country, as well as being the country with the highest SRB for the longest elapsed period and the country experiencing the most profound

Mengjun Tang

mengjuntang@vip.sina.com

1 China Population and Development Research Center, Beijing, China 
impacts from distorted SRB. As such, China's practices in addressing skewed SRB could be a valuable reference for other countries.

This paper reviews the causes of skewed sex ratio at birth and the changing trend in China, summarizes governance practices to address the issue, and discusses the challenges China faces looking forward.

\section{Changes in sex ratio at birth}

The SRB of China was first observed to be abnormal in 1982. A ratio of 108.47 in that year increased rapidly to a peak of 121.18 in 2004, after which SRB has fluctuated around $120 .{ }^{1}$ Since 2009 , the SRB has declined slowly but steadily, from 120.56 in 2008 to 110.14 in 2019. Although China's SRB remains skewed today, indicating the demographic masculinization of China's population over the long term, the overall trend is expected to continue declining and will get close to normal level in the next ten years.

Gender imbalance at birth in China, initially an issue in several provinces, spread to cover the entire country, and from rural to urban areas. Among China's 32 provinces, 19 had normal SRB in 1982, while only one did in 2015. Most provinces have had or are experiencing gender imbalance at birth to varying degrees. There were still 3 provinces with extremely skewed ratios above 120 in 2015 . Several provinces in the central and southeastern regions are the main contributors to the imbalanced SRB of China's total population.

The masculinization of births first appeared in rural areas and spread to towns and cities where social-economic conditions were relatively developed. In recent years, the gap between SRB in rural and urban areas has narrowed, and towns have replaced rural areas to become the worst-hit areas with the most distorted SRB.

In China, SRB in affected populations inevitably rise with birth order, as parents react to the absence of prior male births. In 1982, the SRB for first and second children was normal, but for three or more children it reached 127, much higher than normal. By 1990, the SRB for second children and for three or more children had become skewed, and in 2010, even the SRB for first children was abnormal. This indicates that prenatal sex selection became more likely as the number of births increased. In 2015, the SRB of different parities had decreased significantly, with SRB of the first child, though still on the high side, having fallen below 110. The most significant decrease was observed in the sex ratio of the second child.

Gender imbalance at birth is affected by a combination of social, economic, cultural, demographic, and other factors. Objectively speaking, an effort to have sons is a rational strategy to respond to fertility decline for couples living in a maleoriented patriarchy. In the context of a restrictive fertility regime, couples adapt by seeking fetal sex determination and sex selection to realize their preference for sons. An urgency imposed by reduced family fertility, a strong preference for sons, and

\footnotetext{
1 The data source of all SRB data quoted in this paper is China National Bureau of Statistics, Population Census, the $1 \%$ National Population Sample Survey.
} 
the availability of sex selection technology are three indispensable preconditions, which together determine the emergence of skewed SRB and the extent to which it is skewed (Guilmoto 2009). In addition, the underreporting of female births inflates this imbalance in the statistics.

\section{China's practice of addressing the skewed SRB}

The rise and decline of the SRB in China coincides with the country's rapid socioeconomic development during the past several decades. Active governance measures addressing SRB together with rapid socio-economic development are the main driving forces behind the decline of SRB.

First, over time socio-economic development has increased incomes, spread the concept of gender equality, and modernized lifestyles. Improved access to education and employment opportunities among younger cohorts of women have empowered women and increased female autonomy. These socio-economic factors have caused the preference for sons to diminish gradually. Second, effective government interventions have accelerated the decline of SRB. The following focuses on government interventions and governance measures.

\subsection{Four stages of governance}

During the past several decades, government's awareness of SRB has increased and interventions have made steady progress. Government activities can be divided into four stages, each with a different focus:

\subsubsection{Identification of masculinization and the initiation of research (1982-1995)}

Chinese scholars found that the underreporting of female births rather than female infanticide was the main cause of birth masculinization in the 1980s, and that gender-biased sex selection in favor of sons has been the main driver since the 1990s (Zeng et al. 1993). Not only was there prenatal sex selection, but the mortality rate of girls aged 0-4 years was also abnormally high in the 1990s because of discrimination against girls ( $\mathrm{Li}$ et al. 1999). Studies reveal that the preference for sons is strongly related to gender inequality that advantages male children at the expense of female children, and is the root cause of the skewed SRB. (Tang 2008; Chin and Ye 2011; Zhuang 2006).

\subsubsection{Initial pilot project, and information, education and communication (IEC) activities at the national level (1996-2003)}

In response to the grave problem represented by a continuing rise in the sex ratio at birth, and also influenced by the 1995 World Women Conference, the Chinese government launched the "Campaign to Bring New Concepts of Marriage and Childbearing to Families" in 1998. A key component of this campaign was to promote the concept 
of gender equality in marriage and childbearing. From 2000 to 2002, a pilot project for "Improving the Living Environment for Girls" was carried out in Chaohu City, Anhui Province. The objectives of this effort were to improve the living environment of girls and explore local experiences in balancing SRB.

\subsubsection{Expanding the scope the pilot project: campaign of Care for Girls (2003-2013)}

In 2003, based on the success of pilot project in Chaohu City, the National Population and Family Planning Commission selected 24 counties/districts with the highest SRB in 24 provinces and carried out a larger scale pilot project called "Care for Girls". This project advanced a " $5+1$ " governance model that aimed to curb a further rise of SRB by creating a supportive environment for the survival and development of girls. In this model, "1" referred to strengthening leadership, and " 5 " referred to strengthening IEC activities and the advocacy of gender equality, strictly banning the "two illegalities" (illegal fetus sex determination and illegal sex selective induced abortion), providing integrated prenatal services, formulating policies favorable to girls, and improving birth statistics. Monitoring and evaluation reports for the 24 pilot counties, released in 2005 , showed that SRB in the counties demonstrated an overall downward trend after three years of intervention (Xi and Chen 2005).

In 2005, the General Office of the State Council forwarded a comprehensive Action Plan jointly issued by 12 government departments to address abnormally high SRB. The plan called for expansion of the campaign to "Care for Girls" into a national strategic initiative. The campaign was implemented nationwide and given a high priority in the government's agenda. In 2006, the government established strategic goals and plans for SRB levels from 2006 to 2020, dividing the years into three five year periods, each with a strategic target. The goal of the first five year period was to curb SRB growth, of the second to bring about a decline of SRB, and finally the third period targeted SRB stabilization at a normal level.

\subsubsection{Stability and consolidation of governance ( 2013 to date)}

During this period, governance and government interventions have remained stable, with an emphasis on closer cooperation with medical institutions to strengthen the crackdown on the "two illegalities." Comprehensively addressing the skewed SRB was included in the National Population and Family Planning Development Plan for implementation during China's 13th Five-year Plan (2016-2020). The goal of normalizing SRB has been incorporated into "The Outline of the 2030 Plan for Healthy China" and "The National Population Development Plan (2016-2030)", two program documents for the country's development. 


\subsection{Practical measures to address imbalanced sex ratio at birth}

\subsubsection{IEC campaign targeting the general public to weaken the preference for sons and advocacy targeting policy-makers to formulate policies with increased sensitivity to gender concerns}

This campaign employed a variety of promotional approaches, including the use of posters, slogans, television, the Internet and other media channels, as well as innovative approaches designed to change the customs and traditional practices that shaped a couple's preference for sons. Cultural sensitivity was taken into account.

\subsubsection{The empowerment of women and supportive policies for girls' families}

The Chinese government developed policies and formulated laws to protect women's rights and interests, and implemented the "Outline for the Development of Chinese Women" to promote the development of women in the economy, education, health, social security, and the environment, and their participation in decision-making processes. Local governments have introduced a number of policies that give priority to families with only daughters in terms of schooling, employment, economy, old-age care, land, housing and so on.

\subsubsection{Measures to control accessibility to sex selection technology}

In response to China's imbalanced SRB, in 1986 the National Family Planning Commission and the Ministry of Health jointly issued the "Notice on Prohibition of Arbitrary Fetal Sex Determination", followed by the "Law of the People's Republic of China on Maternal and Infant Care" in 1994, and the "Population and Family Planning Law of the People's Republic of China" in 2001. These measures made fetal sex determination and sex-selective pregnancy termination for non-medical purposes illegal. The government also strengthened the education of medical personnel, severely punished those engaged in the "two illegalities", and regulated the use of fetal sex determination and selection technology and drugs. Termination of pregnancies more than 14 weeks after conception for non-medical purposes was strictly limited.

\subsubsection{Improvement in the quality of birth statistics to ensure the reliability and accuracy of birth data}

After 2000, birth records were computerized, and this has helped to reduce misreporting and errors in statistics. In recent years, independent information systems have been linked, and data sharing and comparison system have been established, further improving the accuracy of birth data. 


\section{Conclusion and discussion: experiences and challenges}

In 2011, OHCHR, UNFPA, UNICEF, UN Women and WHO issued an interagency statement on the prevention of gender-based fetal sex selection (OHCHR, UNFPA, UNICEF, UN Women and WHO 2011). The statement recommended five important interventions to combat skewed SRB: provision of reliable data and research, appropriate use of technology, supportive policies for girls and women, policies and laws to promote gender equality, and extensive communication, advocacy and community mobilization. An examination of the record shows that the practices China has employed to address skewed SRB are highly consistent with the recommendations proposed by the international community, while at the same time they have, without doubt, distinct Chinese characteristics.

The Chinese government has strong leadership and executive capabilities, both of which make it possible for the government to mobilize resources effectively to achieve its goals. The government has taken all-around measures to address the problem of gender imbalance at birth. At both the macro and micro levels, changes to lifestyles and values brought about by socio-economic development and modernization have undoubtedly been a great driving force for the weakening of the preference for sons. A holistic governance framework has been adopted in China's governance model for SRB (Li et al. 2013), with multi-sectoral involvement and consistent governance approaches at all administrative levels. The policies and measures adopted are comprehensive and multi-faceted, not only aiming at the root causes of son preferences and improving the welfare of girls' families, but also at reducing occurrences of the "two illegalities" that are a direct cause of birth masculinization. The government has also strengthened policy implementation in those provinces with higher SRB and taken targeted measures that reflect unique local conditions.

China's SRB is, nonetheless, still imbalanced after more than 30 years of governance. The downward trend is a positive sign, but the current level is still abnormal, and this is not a cause for optimism. Looking forward, China's effort to reduce $S R B$ to a normal level still faces many challenges.

- Disparities in regional development in China make it impossible for the country's SRB to decline in the short term. China is a country with a diverse culture and great regional variations in terms of socio-economic development. This means that the decline of SRB exhibits regional disparities and is not synchronous nationally. It is estimated that the SRB in some regions will remain abnormal for a longer time, resulting in a slower decline of the SRB at national level. There is still a long way to go to get the ratio back to the pre1982 level.

- Gender inequality is difficult to eliminate in a short period of time. China's historical tradition of patriarchy is still omnipresent. The concept of gender equality is not fully reflected in public policies. Although gender discrimination is rarely embodied literally in any official or legal documents in China; in reality, unwritten or implicit gender discrimination can be found somewhere. 
It is more difficult for female college graduates to get a job than it is for male graduates, and there is still a considerable gap in income between men and women. The political participation of women at the grassroots level is not satisfactory, and the proportion of women in senior leadership positions is disproportionately low. Women are often responsible for most housework in the family, and bear most of the burden of childbearing and childrearing. Such gender inequalities are rooted in tradition, culture and institutions, and cannot be easily or quickly eliminated. This means that the preference for sons will persist well into the future. Promoting gender equality and narrowing the gender gap in areas like employment and income are fundamental to weakening son preference and thus normalizing the SRB.

- The advancement of technology makes gender-biased sex selection much easier to accomplish and more difficult to detect. Today, new technologies are able to accurately identify the sex of the fetus before the 14th week of pregnancy. Sex-selection technologies combined with limited restrictions on abortion make it much easier than in the past for Chinese couples with strong son preference to resort to gender-based pregnancy termination. In the long run, banning sex selection technology will become increasingly costly and difficult.

- The gender imbalance at birth is closely connected with low fertility, changes in family structure, the aging of society, urbanization, and numerous other issues. Together, these factors impose significant negative costs on social security and socio-economic development. China will have a gender imbalanced society for the next 40 years. Over the long-term, skewed SRB leads to a large "shortage" in the number of women. Women play an irreplaceable role in the family and society. A long-term and large-scale shortage of women not only aggravates the marriage squeeze men face, but also results in a reduction in the size of the female population of reproductive age. As China's population ageing, the need for elderly care is increasing, and the shortage of women will present a formidable challenge to satisfying the demand for care because the majority of elderly care providers are women.

- There is an urgent need to strengthen international cooperation in the governance of gender imbalances. Gender imbalance is not solely a phenomenon in China, but a common problem in a number of other countries and regions. Although the causes and trends of gender imbalances are not the same in all countries and regions, the consequences of gender imbalance are very similar from place to place. Moreover, the effects of these consequences cross national borders at will and can last for one or two generations. In the broader context of globalization, as the number of transnational marriages in China has increased, issues such as the legitimacy of marriage, marriage fraud, the protection of married people's rights and their social integration have recently emerged. In the face of such complex challenges that will be with us well into the future, it is necessary to consolidate a conceptual consensus for community that embodies a shared future for humankind and, on that basis, to build a global governance framework for gender imbalance. 


\section{Compliance with ethical standards}

Conflict of interest The author has no potential conflicts of interest.

Open Access This article is licensed under a Creative Commons Attribution 4.0 International License, which permits use, sharing, adaptation, distribution and reproduction in any medium or format, as long as you give appropriate credit to the original author(s) and the source, provide a link to the Creative Commons licence, and indicate if changes were made. The images or other third party material in this article are included in the article's Creative Commons licence, unless indicated otherwise in a credit line to the material. If material is not included in the article's Creative Commons licence and your intended use is not permitted by statutory regulation or exceeds the permitted use, you will need to obtain permission directly from the copyright holder. To view a copy of this licence, visit http://creativecommons.org/licen ses/by/4.0/.

\section{References}

Guilmoto, C. Z. (2009). The sex ratio transition in Asia. Population and Development Review, 35(3), 519-549.

Li, S., Zhu, C., \& Han, S. (1999). Analysis of 0-4 child mortality in Shaanxi Province, 1994-1996. Chinese Journal of Population Science, 2, 43-52. (in Chinese).

Li, S., Shang, Z., \& Feldman, M. W. (2013). Social management of gender imbalance in China: A holistic governance framework. Economic \& Political Weekly, 48(35), 79-86.

OHCHR, UNFPA, UNICEF, UN Women and WHO. (2011). Preventing Gender-biased Sex Selection: An Interagency Statement. Geneva: WHO.

Tang, Z. (2008). Skewed sex ratio at birth from gender perspectives. Population Journal, 4, 15-19. (in Chinese).

Xi, X., \& Chen, S. (2005). Evaluation of Care for Girl Campaign. Beijing: China Population Press. (in Chinese).

Zeng, Y., Tu, P., Gu, B., Xu, Y., Li, B., \& Li, Y. (1993). Causes and implications of the recent increase in the reported sex ratio at birth in China. Population and Development Review, 19(2), 283-302.

Zhuang, Y. (2006). Hierarchic analysis of women's low status-underlying causes of skewed sex ratio at birth in China. Southern Population, 1, 41-50. (in Chinese).

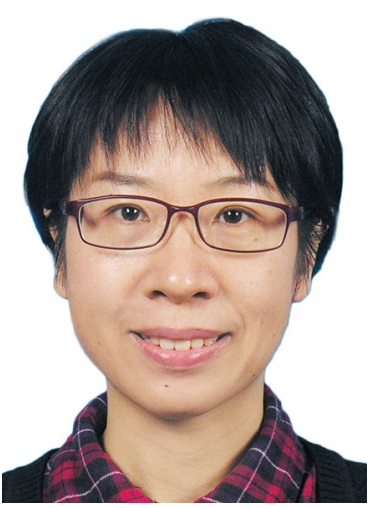

Mengjun Tang is a senior research fellow in China Population and Development Research Center. 